\title{
Is there any Sympathetic Skin Response Abnormality in Raynaud Phenomenon?
}

Mohammad R. Emad, ${ }^{1}$ "Hamid R. Farpour, ${ }^{1,2}$ Faisal Ahmed, ${ }^{3}$ Masoumeh Tayebi, ${ }^{1}$ Mohammadali Nazarinia, ${ }^{2,4}$ Mohammad R. Askarpour, ${ }^{5}$ Hossein-Ali Nikbakht ${ }^{6}$

ABSTRACT: Objectives: This study aimed to evaluate sympathetic skin response (SSR) among patients with Raynaud phenomenon (RP). SSR is a technique for assessment of the damage of peripheral neuropathies and the disorders of the sympathetic system. Methods: Between January 2015 and December 2018, approximately 20 patients with RP and 20 healthy subjects (control group) were recruited from patients referred to the outpatient clinics of Shiraz University of Medical Sciences, Shiraz, Iran. All participants were clinically examined and the SSR was evaluated using a standard protocol. SSR is abnormal when the latency is prolonged and/or the amplitude is reduced. Results: The RP group consisted of 19 women (95\%) and one male (5\%); three patients (15\%) had primary Raynaud's phenomenon (PRP) and 17 patients (85\%) had secondary Raynaud's phenomenon. The control group consisted of 16 women (80\%) and four males (20\%). The mean age of the RP group and control subjects was 43.1 \pm 9 and $36.7 \pm 8.6$ years, respectively. The SSR to the electrical stimulus was absent in three patients with PRP. The total median nerve mean latencies in the upper limb were $1.90 \pm 0.57$ and $1.19 \pm 0.52$ seconds for the RP group and control groups, respectively $(P<0.001)$. These findings revealed significantly prolonged SSR latencies in the RP group, while the mean amplitude showed no significant differences in both groups $(P=0.756)$. Conclusion: Absence or prolonged latency of SSR was associated with the disorders of the unmyelinated axons in the sympathetic system. The findings of the present study suggested the disorders of unmyelinated axons in Raynaud's phenomenon.

Keywords: Raynaud Disease; Autonomic Nervous System; Electrodiagnosis; Sympathetic Fibers; Nerve Conduction; Iran.

\section{Advances IN KNOWLedge}

It is expected that this study, conducted in Iran, will increase both individual and physicians' awareness of Raynaud's phenomenon (RP).

The absence or prolonged latency of the sympathetic skin response was associated with disorders of unmyelinated axons in the sympathetic system such as RP.

\section{Application to Patient Care}

The sympathetic skin response test is recommended to be used for evaluating the sympathetic status and symptoms in patients with RP.

$\mathrm{R}$ AYNAUD PHENOMENON (RP) IS A CONDITION in which a sequence of pallor, cyanosis and redness manifest when one is exposed to cold weather or stress. This condition can be painful and lead to recurrent vasospasms in the digits. ${ }^{1,2}$ There are two types of RPs: primary Raynaud's phenomenon (PRP) and secondary Raynaud's phenomenon (SRP). The criteria of PRP includes a symmetric presentation of this phenomenon without tissue necrosis, ulceration or gangrene after secondary causes are ruled out. ${ }^{3}$ SRP occurs in association with underlying diseases, including neurological disorders and mixed connective tissue disorders. ${ }^{3}$ Sympatholytic drugs and reduction of emotional stress are useful in RP treatments; it has been suggested that autonomic nervous system (ANS) dysfunctions may lead to RP. ${ }^{1}$ To evaluate a patient presenting with RP, a physician should obtain the required laboratory data and imaging after acquiring the patient's medical history and completing their physical examination. These assessments are essential tools in the broader evaluation of RP symptoms. ${ }^{4}$ To manage the phenomenon, patients should consider lifestyle modification, keep the affected areas warm and stop taking vasoconstricting drugs such as nicotine. Hughes et al. reported that only $16 \%$ of the participants with RP stated that at least one current medication had been effective to prevent or control RP attacks. $^{5}$

Sympathetic skin response (SSR) is evaluated using a simple and non-invasive test that reveals an interaction between the surrounding epidermal tissue and sweat glands. ${ }^{6}$ Because it is a multisynaptic reflex, its waveform amplitude and latency are variable. SSR evaluation involves recording the electrical stimulus of a peripheral nerve from the surface electrodes attached to the hands and feet in order to study the neural activity of type $\mathrm{C}$ unmyelinated fibres. It is a reliable indicator of the sudomotor sympathetic 
function, used to evaluate patients with somatic and autonomic neuropathies. ${ }^{6,7}$

Most of the recent studies have assessed the large myelinated fibres and not measured the thin myelinated or unmyelinated fibres including the sympathetic fibres within the peripheral nerve. ${ }^{8}$ There are few studies that evaluate the ANS effect electrophysiologically in patients with RP. This study evaluates the SSR in patients with RP to assess the sympathetic dysfunction found in such patients.

\section{Methods}

The present study included 20 patients with PRP and SRP in the period between January 2015 and December 2018. The patients were recruited from those referred to the outpatient clinics of Physical Medicine \& Rehabilitation and Rheumatology departments of Shiraz University of Medical Sciences, Shiraz, Iran. The control group included 20 healthy participants who did not consume any medication affecting the ANS. Written informed consent was taken from all participants to be enrolled in the present study.

Patients included in this study were those who had been diagnosed with PRP and SRP according to previously published criteria and had definite sensitivity to cold with the classic colour sequence triad in their hands. ${ }^{9}$ Patients were selected at least six months after the onset of the symptoms and were recruited from among those referred to the outpatient clinics of the physical medicine and rehabilitation and rheumatology departments of the university.

Patients were excluded if they had been diagnosed with endocrine diseases such as diabetes mellitus, thyroid diseases and metabolic diseases. Patients with scars, ulcers or gangrene of the fingers, neurological disorders, other mixed connective tissue diseases, a history of cancer or any type of peripheral neuropathy were excluded as well. Furthermore, patients were also excluded if they used drugs that are known to affect the ANS (e.g. tricyclic antidepressants, clonidine, ergotamine and serotonin-receptor agonists, B-blockers or oral contraceptives).

At first, general clinical and neurological examinations were performed for all subjects in a half dark and silent room with a temperature of 23-26 ${ }^{\circ} \mathrm{C}$ and a humidity between $30-35 \% .{ }^{10}$ SSRs with an intensity between 15-25 mA were used to assess the sympathetic activity in 20 patients with RP and 20 controls. The skin temperature was kept above $32{ }^{\circ} \mathrm{C}$ in all patients. One electromyographic apparatus, the Medelec Synergy electromyograph (CareFusion Corp., San Diego, California) was used for all patients and the control group by the same physiatrist. ${ }^{10}$ A sweep speed of $500 \mathrm{~ms} /$ div, sensitivity of $200-1000 \mathrm{mV} / \mathrm{div}$ and filtering of $0.5 \mathrm{kHz}$ were used. ${ }^{11}$

The subjects were conscious, silent and fixed in a supine position to diminish movement artifacts. Before performing the test, the procedure was explained to all the participants. For the median nerve SSR, the active electrode was attached to the base of the second finger in the palmar surface and the reference electrode was placed in the dorsal aspect of the hand. Stimulating electrodes were placed on the wrist area between the palmaris longus and flexor carpi radialis tendons. A ground electrode was located proximal to the active electrode with respect to the cathode's location [Figures 1 and 2]. ${ }^{10}$

To record the SSR, the authors used the minimum stimulation intensity which was needed and increased it in the subsequent stimulation. The stimuli were given at irregular intervals of more than 65 seconds to avoid considerable habituation. Latencies and amplitudes of the waves were measured. The latency of SSR was measured in seconds from the stimulation artifact onset to the onset of the first deflection from the baseline; the amplitude was measured in $\mu \mathrm{V}$ from peak to peak (i.e. the peak of the first deflection to that of the next one). Three recordings were performed for each limb and the average responses were used for analysis. ${ }^{12}$ An absent response was considered an abnormal test. Median and tibial nerve compound muscle action potential (CMAP) and median and sural sensory nerve action potential (SNAP) were done for all subjects for assessment of any peripheral neuropathy (e.g. carpal tunnel syndrome). Only the reproducible responses with no movement artifacts were chosen for analysis. ${ }^{13}$

Statistical Package for the Social Sciences (SPSS), Version 18 (IBM Corp., Chicago, Illinois, USA) was used to analyse the data. Arithmetic mean and standard deviation of the data were determined using a T-test and Mann Whitney's U test. The Chi-square test was utilised to compare the responses from the RP and control groups. $P$ values less than 0.05 were considered significant.

This study was approved by the ethics committee of Shiraz University of Medical Sciences (IR.SUMS. REC. 1387.S4254).

\section{Results}

The present study consisted of 20 RP patients, 19 (95\%) of whom were females. The patients' mean age was $43.1 \pm 9$ years. The control group consisted of 16 females (80\%) and four males (20\%). The mean age of the control group subjects was $36.7 \pm 8.6$ years. No underlying diseases were found in three patients (15\%) 


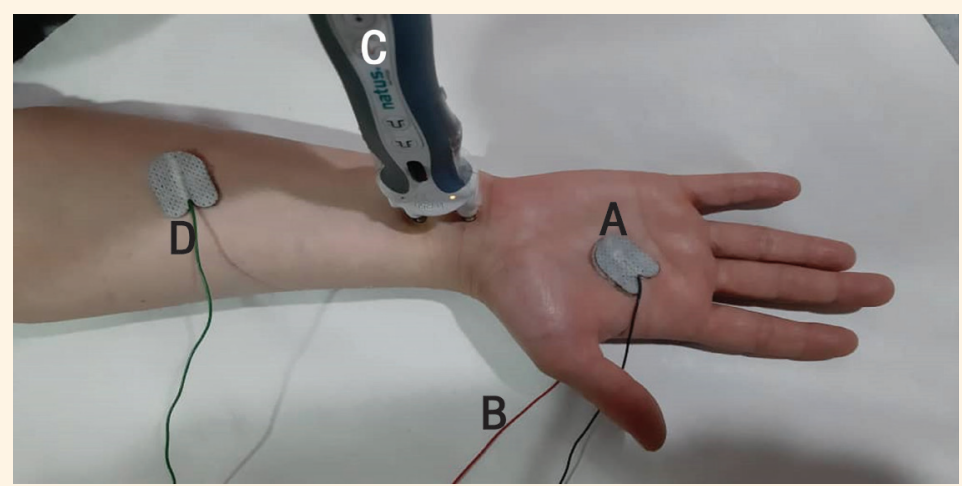

Figure 1: Photograph showing the sites at which sympathetic skin response from the median nerve were recorded using an electromyograph. Sites included an active electrode on the hand (A), a reference electrode on the dorsal aspect of the hand (B), a stimulating electrode for the median nerve on the wrist (C) and a ground electrode on the forearm (D).

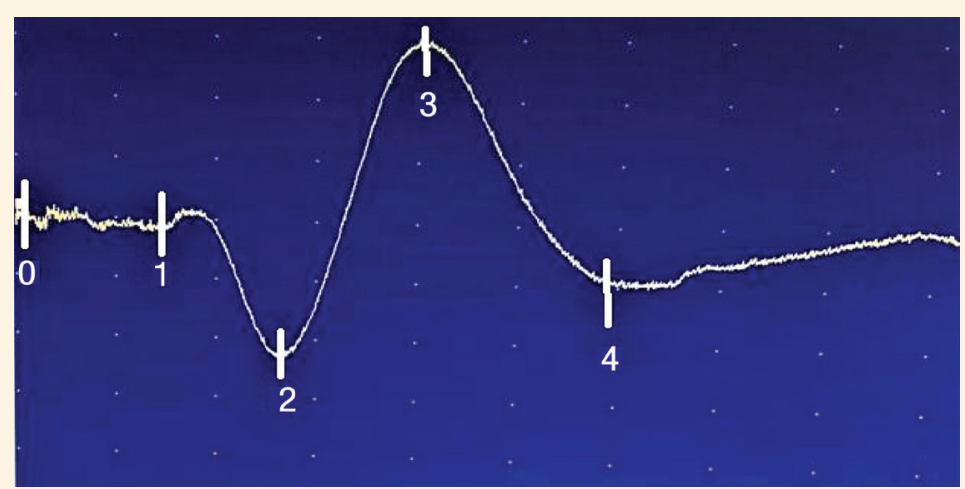

Figure 2: Photograph of the sympathetic skin response waveform showing onset latency in seconds (range: 0-1), peak to peak amplitude in microvolts (range: 2-3) and the total duration of the sympathetic skin response in seconds (range: 1-4).

Table 1: Comparison of parameters of the sympathetic skin response between the Raynaud's phenomenon (RP) and control groups $\left(\mathrm{N}_{\mathrm{RP}}=20 ; \mathrm{N}_{\mathrm{C}}=20\right)$

\begin{tabular}{|c|c|c|c|}
\hline Parameters & $\begin{array}{c}\text { RP group } \\
(\text { mean } \pm \text { SD) }\end{array}$ & $\begin{array}{c}\text { Control group } \\
(\text { mean } \pm \text { SD })\end{array}$ & $P$ value \\
\hline \multicolumn{4}{|c|}{ Latency in seconds } \\
\hline $\begin{array}{l}\text { Right } \\
\text { median } \\
\text { nerve }\end{array}$ & $1.86 \pm 0.55$ & $1.11 \pm 0.50$ & $*<0.001$ \\
\hline $\begin{array}{l}\text { Left median } \\
\text { nerve }\end{array}$ & $1.79 \pm 0.49$ & $1.21 \pm 0.65$ & $*<0.002$ \\
\hline Upper limbs & $1.90 \pm 0.57$ & $1.19 \pm 0.52$ & $* 0.001$ \\
\hline \multicolumn{4}{|c|}{ Amplitude in mV } \\
\hline $\begin{array}{l}\text { Right } \\
\text { median } \\
\text { nerve }\end{array}$ & $1.14 \pm 0.89$ & $0.93 \pm 0.48$ & 0.358 \\
\hline $\begin{array}{l}\text { Left median } \\
\text { nerve }\end{array}$ & $0.85 \pm 0.5$ & $1.01 \pm 0.50$ & 0.318 \\
\hline Upper limbs & $0.94 \pm 0.7$ & $1.00 \pm 0.50$ & 0.756 \\
\hline
\end{tabular}

with PRP. However, the remaining 17 patients (85\%) with SRP had a history of underlying diseases such as systemic sclerosis $(\mathrm{n}=14 ; 70 \%)$ and rheumatoid arthritis (RA; $\mathrm{n}=3 ; 15 \%$ ). All of the PRP patients had abnormal sympathetic SSR latency and amplitude tests (100\%).

The mean latencies for SSR of the right and left median nerves lasted $1.86 \pm 0.55$ and $1.79 \pm 0.49$ seconds in the RP group and $1.11 \pm 0.50$ and $1.21 \pm$ 0.65 seconds in the control group, respectively. The total median nerves mean latencies in the upper limb was $1.90 \pm 0.57$ and $1.19 \pm 0.52$ seconds for the RP and control groups, respectively. Comparisons in both groups demonstrated significant differences for SSR mean latencies $(P<0.001)$. However, the comparison of SSR mean amplitudes in both groups did not show a significant difference $(P=0.756)$ [Table 1 ].

All individuals had normal median and tibial nerve CMAP and median and sural SNAP. Any peripheral neuropathy such as carpal tunnel syndrome or peripheral polyneuropathy was excluded.

\section{Discussion}

The present study aimed to investigate electrophysiologic alterations of the sympathetic 
nervous system in RP patients. There are some conditions associated with abnormal SSR such as lesions of the peripheral nerves as well as those of the nerve roots (diabetic neuropathy, familial amyloid neuropathy, alcoholic neuropathy, lepromatous neuropathy). ${ }^{8}$ Additionally, abnormality of SSR was observed in carpal tunnel syndrome and complex regional pain syndrome. ${ }^{7,14}$

Measures have been taken to utilise the SSR in the diagnosis of sympathetic damage in some rheumatologic disorders such as scleroderma, Sjogren's syndrome, RA, fibromyalgia, chronic fatigue syndrome and RP and in urologic diseases such as chronic prostatitis. ${ }^{11,15-17}$ To the best of the authors' knowledge, the literature lacks adequate data regarding ANS evaluation in patients with RP. Neurological changes in RP are doubtful.

When used clinically, SSR has many limitations. Latency and amplitude of the signal response are different in a single individual and even more diverse when studying a population. Adding the habituation phenomenon to letters makes it challenging to calculate normal SSR parameters. ${ }^{18,19}$ One study on the clinical use of SSR reported lower amplitude response in the contralateral side than the side of common peripheral nerve stimulation. ${ }^{19}$ This might happen due to greater excitation dispersion of the afferents arc. When studying the role of sympathetic fibres in mononeuropathies, one can bypass the obtaining absolute reference values dilemma using the unaffected side as an internal control by comparing the parameters between the two sides. Thus, the ratio of values between the two side values can be calculated. ${ }^{18,19}$ Based on previous studies, to identify the abnormal response in the amplitude and latency of SSR, the present study made a comparison between patients and normal individuals.

The results of the present study showed that PRP patients showed abnormal SSR latency and amplitude, which was similar to a previous study carried out by Mondelli et al. ${ }^{20}$ The authors reported sympathetic dysfunction in PRP. While they stimulated the ulnar nerve in the upper limb, the authors of this study stimulated the median nerve that has more sympathetic fibres and found a significant delay in SSR latency in the patients with PRP. ${ }^{20}$ Additionally, instead of SSR amplitude, this study measured the area due to good accuracy of values for total sympathetic excitability of the nerve.

According to a study carried out by Charkoudian, the sympathetic nervous system, via both peripheral (local) and central system mechanisms, plays a critical role in the pathogenesis of PRP and SRP. ${ }^{21}$ Pancera et al. investigated the sympathetic hyperactivity in systemic sclerosis and PRP. ${ }^{15}$ Subjects with PRP had normal heart rate changeability and more activity of sympathetic fibres. In another study, PRP was compared with SRP in systemic sclerosis. Both groups showed a decrease in calcitonin gene-related peptide, endothelium-1 flare and a prostaglandin production value of 9.5. These findings indicate that there is a general vascular hyperactivity in these conditions that probably reflect a primary vascular disorder. ${ }^{22}$

In a study on 20 patients with SRP, Gosk-Bierska et al. noted a significantly low SSR amplitude and long latency in their palms and soles. ${ }^{23}$ In patients with SRP, no relationship was found between SSR and microangiopathy; this confirmed that these two processes occur independently in patients with SRP. The authors concluded that normal peripheral nerve function with impaired ANS suggested the central origin of SRP. Abnormal SSR habituation might also result from the central mechanism. ${ }^{23}$ Gledhill et al. showed ANS dysfunction in nine SRP patients with cardiovascular autonomic dysfunction. ${ }^{24}$ The heart rate response to deep breathing-Valsalva and standingwere measured before and after triiodothyronine administration. The amplitude was decreased in three patients, and mild slowing of conduction velocity in six patients (less than 20\%) was observed. Test results showed considerable improvement of ANS function after administration of triiodothyronine. ${ }^{24}$

Using median nerve stimulation, Badry et al. compared the SSR in 21 patients with systemic sclerosis (SSc) and 39 patients with RA to SSR in 60 healthy participants. ${ }^{25}$ They found increased latency and reduced amplitude in SSc and RA patients. The SSR of SSc patients was significantly prolonged in latency and showed reduced amplitude when compared to RA patients. Additionally, six SSc patients had increased SSR latency without manifestations of polyneuropathy. The authors concluded that patients with SSc and RA suffered from ANS dysfunction with more effects being seen in SSc patients. ${ }^{25}$ Saba and Sultan evaluated the ANS changes in RA patients. The SSR amplitude decreased and latency was prolonged in RA patients compared to the control group. Additionally, there were no statistically significant differences between the patients with different disease activity of RA and functional disability of RA, SSR latency and amplitude. ${ }^{26}$ Another study of SSR in 30 RA patients showed that SSR was abnormal in six patients. ${ }^{27}$ The authors did not include amplitude as a diagnostic criterion as it was too variable even within the same patient. They found frequent abnormalities in SSR in patients with RA regardless of whether or not there was a clinical symptom of ANS. ${ }^{27}$ 
The findings of the present study are in line with those of previous studies; there was no significant abnormality in SSR amplitude between the patients and controls. Most studies did not consider amplitude as a valid measure of normality versus abnormality. ${ }^{20,27}$ There was also no relationship between the type of SSR abnormality and intensity of autonomic impairment. In one study on reflex sympathetic dystrophy, the mean amplitude and onset latency of SSR in the involved limb was greater and shorter than that of the uninvolved limb. ${ }^{7}$ These findings support the crucial role of ANS dysfunction in RP. ${ }^{28}$ Overall, in the present study, the individuals in the RP group presented with abnormal SSR in regards to the mean latency, suggesting a significant difference between the groups in skin innervation.

In pathophysiology, lack of SSR represents the failure of the polysynaptic system in propagating impulses to the end organs. Increased latency in SSR may originate from milder neuropathic damage or from loss of thicker axons, changes in cholinergic fibres and/or sweat glands and synaptic transmission interruption through central processing. ${ }^{23}$ Since there was no evidence of afferent somatic fibres or central nervous system or sweat gland diseases in the present study, it is suggested that the source of SSR latency abnormalities might be the sympathetic efferent nerves.

Notably, PRP is more common in women than men; thus, many published studies failed to match gender in the control and case groups. ${ }^{20}$ By matching gender between the case and control groups, the authors of this study have tried to control for this bias.

Performing the SSR test in rheumatology patients helps detect autonomic disorders early, thus enabling the patient to receive the appropriate treatment for potential complications (for example, cardiovascular or skin disorders such as dry skin or excessive sweating or, alternatively, neurological disorders such as dizziness and imbalance). ${ }^{27}$ It could also be beneficial in regulating the drugs that affect the autonomic system in RP patients. Furthermore, using the SSR test for better management of underlying autonomous system-related symptoms helps the patient in controlling RP symptoms optimally. ${ }^{20}$ ANS dysfunction is one of the aetiological factors linked to the development of microvascular manifestations of RA and SSc, which may play a role in developing the pain symptoms associated with these diseases. ${ }^{7}$

This study had some limitations including a small sample size $(\mathrm{n}=20)$. This was also just an observational report which would require validation with randomised criteria and a larger sample size. Moreover, some patients with SRP use anti-hypertensive drugs and the authors could not discontinue the medication for the patient group. It is possible that medications could alter the SSR since the test is very sensitive and requires complete relaxation during the examination. Hence, there is a need to implement certain specific techniques in order to account for such confounding variables and enhance patient cooperation. The authors recommend further studies with a larger sample size and clinical trials to better evaluate SSR parameters.

\section{Conclusion}

Absence or prolonged latency of sympathetic skin response was associated with unmyelinated axon disorders in the sympathetic system. The findings of the present study confirm the role of the disorders of unmyelinated axons and sympathetic nervous system in RP.

\section{ACKNOWLEDGEMENTS}

The authors would like to thank the Research Consultation Center (RCC) of Shiraz University of Medical Sciences for their invaluable assistance in analysing the data. The authors would also like to thank the Center for Development of Clinical Research of Nemazee Hospital and Dr. Nasrin Shokrpour for editorial assistance. This study is a part of the thesis of Masoumeh Gerami Tayebi (Grant no: 4254).

\section{CONFLICT OF INTEREST}

The authors declare no conflicts of interest.

\section{FUNDING}

No funding was received for this study.

\section{AUTHORS' CONTRIBUTION}

MA and HF conceptualised the work. MA supervised the work. MT and HF prepared the proposal and collected the data. FA and MN analysed and interpreted the data. MA drafted the manuscript. FA and $\mathrm{HN}$ reviewed and edited the drafted manuscript. HF collected, labelled and formatted the images. All authors approved the final version of the work.

\section{References}

1. Bakst R, Merola JF, Franks AG Jr, Sanchez M. Raynaud's phenomenon: pathogenesis and management. J Am Acad Dermatol 2008; 59:633-53. https://doi.org/10.1016/j.jaad.2008.06.004.

2. Mallipeddi R, Mathias CJ. Raynaud's phenomenon after sympathetic denervation in patients with primary autonomic failure: questionnaire survey. BMJ 1998; 316:438-9. https://doi.org/10.1 136/bmj.316.7129.438. 
3. Wigley FM. Clinical practice. Raynaud's Phenomenon. N Engl J Med 2002; 347:1001-8. https://doi.org/10.1056/NEJMcp013013.

4. Pauling JD, Hughes M, Pope JE. Raynaud's phenomenon-an update on diagnosis, classification and management. Clin Rheumatol 2019; 38:3317-30. https://doi/org/10.1007/s10067-019-04745-5.

5. Hughes M, Snapir A, Wilkinson J, Snapir D, Wigley FM, Herrick AL. Prediction and impact of attacks of Raynaud's phenomenon, as judged by patient perception. Rheumatology (Oxford) 2015; 54:1443-7. https://doi.org/10.1093/rheumatology/kev002.

6. Shahani BT, Halperin JJ, Boulu P, Cohen J. Sympathetic skin response--a method of assessing unmyelinated axon dysfunction in peripheral neuropathies. J Neurol Neurosurg Psychiatry 1984; 47:536-42. https://doi.org/10.1136/jnnp.47.5.536.

7. Kim HI, Yang HE, Kim DH, Park YG. Predictive value of sympathetic skin response in diagnosing complex regional pain syndrome: a case-control study. Ann Rehabil Med 2015; 39:116-21. https://doi.org/10.5535/arm.2015.39.1.116.

8. Kucera P, Goldenberg Z, Kurca E. Sympathetic skin response: review of the method and its clinical use. Bratisl Lek Listy 2004; 105:108-16

9. LeRoy EC, Medsger TA, Jr. Raynaud's phenomenon: a proposal for classification. Clin Exp Rheumatol 1992; 10:485-8.

10. Emmer A, Mangalo S, Kornhuber ME. Augmentation of the sympathetic skin response after electrical train stimuli. Front Neurol 2012; 3:152. https://doi.org/10.3389/fneur.2012.00152.

11. Ozkan O, Yildiz M, Arslan E, Yildiz S, Bilgin S, Akkus S, et al. A study on the effects of sympathetic skin response parameters in diagnosis of fibromyalgia using artificial neural networks. J Med Syst 2016; 40:54. https://doi.org/10.1007/s10916-015-0406-0.

12. El-Badawy MA, El Mikkawy DM. Sympathetic Dysfunction in Patients With Chronic Low Back Pain and Failed Back Surgery Syndrome. Clin J Pain 2016; 32:226-31. https://doi.org/10.1097/ ajp.0000000000000250

13. Emad R, Zafarghasempour M, Roshanzamir S. Sympathetic skin response in incomplete spinal cord injury with urinary incontinence. Ann Indian Acad Neurol 2013; 16:234. https://doi. org/10.4103/0972-2327.112479.

14. Cevik B, Kurt S, Aksoy D, Solmaz V. Sympathetic Skin Response and Boston Questionnaire in Carpal Tunnel Syndrome. Neurophysiology 2016; 48:191-6. https://doi.org/10.1007/s11062-016-9588-4.

15. Pancera P, Sansone S, Presciuttini B, Montagna L, Cerù S, Lunardi C, et al. Autonomic nervous system dysfunction in sclerodermic and primary Raynaud's phenomenon. Clin Sci (Lond) 1999; 96:49-57. https://doi.org/10.1042/cs0960049.

16. Dumitru D, Zwarts MJ. Special nerve conduction techniques. In: Dumitru D, Amato A, Zwarts M, Eds. Electro diagnostic Medicine. 2nd ed. Philadelphia: Hanley \& Belfus, 2001. pp. 249.
17. Eslahi A, Farpour H, Hosseini A, Ahmed F, Chowdhury U, Nikbakht HA. Evaluation of the Sympathetic Skin Response in Men with Chronic Prostatitis: A Case-Control Study. Res Rep Urol 2020; 12:239-45. https://doi.org/10.2147/rru.S253101.

18. Bayrak AO, Tilki HE, Coșkun M. Sympathetic skin response and axon count in carpal tunnel syndrome. J Clin Neurophysiol 2007; 24:70-5. https://doi.org/10.1097/01.wnp.0000239107.10424.fa.

19. Mondelli M, Aretini A, Ballerini M, Vecchiarelli B, Rossi A. Sympathetic skin response. Glabella stimulation may be more useful than peripheral nerve stimulation in clinical practice. Auton Neurosci 2011; 164:101-4. https://doi.org/10.1016/j.aut neu.2011.07.002.

20. Mondelli M, de Stefano R, Rossi S, Aretini A, Romano C. Sympathetic skin response in primary Raynaud's phenomenon. Clin Auton Res 2009; 19:355-62. https://doi.org/10.1007/s10286-0090021-6.

21. Charkoudian N. Skin blood flow in adult human thermoregulation: how it works, when it does not, and why. Mayo Clin Proc 2003; 78:603-12. https://doi.org/10.4065/78.5.603.

22. Sulli A, Soldano S, Pizzorni C, Montagna P, Secchi ME, Villaggio B, et al. Raynaud's phenomenon and plasma endothelin: correlations with capillaroscopic patterns in systemic sclerosis. J Rheumatol 2009; 36:1235-9. https://doi.org/10.3899/jrheum.081030.

23. Gosk-Bierska I, Misterska-Skóra M, Wasilewska M, Bilińska M, Gosk J, Adamiec R, et al. Analysis of peripheral nerve and autonomic nervous system function and the stage of microangiopathy in patients with secondary Raynaud's phenomenon in the course of connective tissue diseases. Adv Clin Exp Med 2018; 27:1587-92. https://doi.org/10.17219/acem/75618.

24. Gledhill RF, Dessein PH, Van der Merwe CA. Treatment of Raynaud's phenomenon with triiodothyronine corrects co-existent autonomic dysfunction: preliminary findings. Postgrad Med J 1992; 68:263-7. https://doi.org/10.1136/pgmj.68.798.263.

25. Badry R, Gamal RM, Hassanien MM, El Hamed MA, Hammam N, El Fawal BM. Sympathetic skin response in patients with systemic sclerosis and rheumatoid arthritis. Egypt J Neurol Psychiatr Neurosurg 2018; 54:38. https://doi.org/10.1186/s41983-018-0044-9.

26. Saba E, Sultan H. Autonomic nervous system changes associated with rheumatoid arthritis: Clinical and electrophysiological study. Egypt Rheumatol 2014; 36:157-63. https://doi.org/10.1016/j. ejr.2014.03.002.

27. Tan J, Akin S, Beyazova M, Sepici V, Tan E. Sympathetic skin response and R-R interval variation in rheumatoid arthritis. Two simple tests for the assessment of autonomic function. Am J Phys Med Rehabil 1993; 72:196-203. https://doi.org/10.1097 /00002060-199308000-00005.

28. Vetrugno R, Liguori R, Cortelli P, Montagna P. Sympathetic skin response: Basic mechanisms and clinical applications. Clin Auton Res 2003; 13:256-70. https://doi.org/10.1007/s10286003-0107-5. 